\title{
Impact des Processus Hydrochimique sur la Qualité des Eaux souterraines de la Ville de N'Djaména-Tchad
}

\section{Kadjangaba Edith,}

Laboratoire de l'Eau et de l'Environnement (LABEEN), Faculté des Sciences Exactes et Appliquées, Université de N,Djamena, N'Djamena,

Tchad, Faculté des Sciences et Techniques, Université de Doba, Doba, Tchad

Djoret Daïra, Doumnang Mbaigane Jean-Claude, Laboratoire de l'Eau et de l'Environnement (LABEEN),

Faculté des Sciences Exactes et Appliquées, Université de N,Djamena, N'Djamena, Tchad

Ndoutamia Guelmbaye Anaclet,

Faculté des Sciences et Techniques, Université de Doba, Doba, Tchad Mahmout Yaya,

Laboratoire des substances Naturelle, Faculté des Sciences Exactes et Appliquées, Université de N,Djamena, N'Djamena, Tchad

Doi: 10.19044/esj.2018.v14n18p162 URL:http://dx.doi.org/10.19044/esj.2018.v14n18p162

Abstract

The problem of access to water in quality and quantity is a major challenge, because it strongly influences the socio-economic conditions of populations in developing countries. The water of the basement of the city of N'Djamena is not sheltered from the risk of pollution. She disposes very little functional wastewater disposal system because the collection networks are almost non-existent, moreover there is a lack of infrastructures for evacuation of household waste, as well as the solid waste treatment units. In addition, under the town of N'Djamena there are two main aquifers located respectively at about 10 and $60 \mathrm{~m}$ depth, and the presence of an intermediate aquifer about $30 \mathrm{~m}$ deep was also noted. Like all urban cities, these aquifers are not immune to the risks associated with human activities. In order to highlight the influence of the hydrochemical process on the groundwater quality of the city of N'Djamena, a study was conducted on a sample of well water and borehole. In total, 20 open wells and 16 boreholes were sampled and analyzed. The $\mathrm{pH}$ and the temperature were measured in situ using the $\mathrm{pH} 323$ / Set B mixed 
electrode and the conductivity using the Lf 318 / Set electrode. It is the same for the alkalinity which is determined by titrimetry, using a HACH / 16900 brand digital titrator. The analysis of major ions was carried out at the Laboratory of Hydrogeology of the University of Avignon. the anions $\left(\mathrm{Cl}^{-}\right.$, $\left.\mathrm{SO}^{2-}{ }^{2}, \mathrm{NO}^{-}\right)$were analyzed using a Dionex ion chromatograph, and the cations $\left(\mathrm{Ca}^{2+}, \mathrm{Mg}^{2+}, \mathrm{Na}^{+}, \mathrm{K}^{+}\right)$using an atomic absorption spectrometer for with a relative uncertainty of $5 \%$. The results of the in-situ parameters have shown that the waters have a $\mathrm{pH}$ which is around 6.85 in the wells and 7.24 in the boreholes and the measured temperature is close to the ambient temperature of the air which is $28^{\circ} \mathrm{C}$. The mineralization of the waters is sometimes high and this results in values of conductivities that can reach 1253 $\mu \mathrm{S} / \mathrm{cm}$ ), especially in the wells. This increase in conductivity is accompanied by an increase in $\mathrm{Cl}^{-}$and $\mathrm{NO}_{3}^{-}$ions in the water. Groundwater has a wide variety of chemical facies, among which mention may be made of calciumbicarbonate-type water, sometimes nitrated, sodium bicarbonate and potassium and nitrate, which may have high chloride contents and bicarbonate and chloride and nitrate calcium or sodium. Principal Component Analysis (PCA) has shown that the main processes influencing groundwater chemistry are hydrolysis of silicates, cation exchange between groundwater and clayey minerals, evaporation and the impact of human activities.

Keywords: Lac Chad basin, hydrochemical, mineralization, well, borehole

\section{Résumé}

La problématique d'accès à l'eau en qualité et quantité est un défi majeur, car elle influe fortement sur les conditions socio économiques des populations de pays en voie de développement. L'eau du sous sol de la ville de N'Djaména n'est pas à l'abri des risques de pollution. Elle dispose très peu de système fonctionnel d'évacuation des eaux usées car les réseaux de collecte sont quasi-inexistants, de plus il y manque les infrastructures pour évacuer les ordures ménagères, ainsi que les unités de traitement des déchets solides. Par ailleurs, sous la ville de N'Djaména on trouve deux principales nappes situées respectivement à environ 10 et $60 \mathrm{~m}$ de profondeur, et la présence d'une nappe intermédiaire à environ $30 \mathrm{~m}$ de profondeur a également été notée. A l'instar de toutes les villes urbaines ces nappes ne sont pas à l'abri des risques liés aux activités humaines. Dans le but de mettre en évidence l'influence du processus hydrochimique sur la qualité des eaux souterraines de la ville de N'Djaména, une étude a été réalisé sur un échantillon des eaux de puits et de forage. Au total : 20 puits à ciel ouvert et 16 forages ont été prélevés et analysés. Le $\mathrm{pH}$ et la température ont été mesurés in situ grâce à l'électrode mixte pH 323/Set B et la conductivité grâce à l'électrode Lf 318/Set. Il en est de même pour l'alcalinité qui est déterminée par titrimétrie, à l'aide d'un titrateur digital de 
marque HACH/16900. L'analyse des ions majeurs a été réalisée au Laboratoire d'Hydrogéologie de l'Université d'Avignon. les anions $\left(\mathrm{Cl}^{-}, \mathrm{SO}_{4}{ }^{2-}\right.$ , $\left.\mathrm{NO}_{3}{ }^{-}\right)$ont été analysés à l'aide d'un Chromatographe ionique Dionex, et les cations $\left(\mathrm{Ca}^{2+}, \mathrm{Mg}^{2+}, \mathrm{Na}^{+}, \mathrm{K}^{+}\right)$à l'aide d'un spectromètre d'absorption atomique pour avec une incertitude relative de $5 \%$. Les résultats des paramètres in-situ ont montré que les eaux ont un $\mathrm{pH}$ qui tourne autour de 6,85 dans les puits et 7,24 dans les forages, et la température mesurée avoisine la température ambiante de l'air qui est de $28^{\circ} \mathrm{C}$. La minéralisation des eaux est parfois élevée et cela se traduit par des valeurs de conductivités qui peuvent atteindre $1253 \mu \mathrm{S} / \mathrm{Cm}$ ), surtout dans les puits. Cette augmentation des conductivités s'accompagne d'une hausse des ions $\mathrm{Cl}^{-}$et $\mathrm{NO}_{3}{ }^{-}$dans les eaux. Les eaux souterraines présentent des faciès chimiques très variables parmi lesquels on peut citer les eaux de type bicarbonaté calcique parfois nitraté, bicarbonaté sodique et potassique et nitraté pouvant avoir des teneurs en chlorures élevées et bicarbonaté et chloruré et nitraté calcique ou sodique. L'analyse en Composante Principale (ACP) a suggéré que les principaux processus influençant la chimie des eaux souterraines sont l'hydrolyse des silicates, l'échange de cations entre les eaux souterraines et les minéraux argileux, l'évaporation et l'impact des activités anthropiques.

Mots clés : Bassin du Lac Tchad, Hydrochimie, minéralisation, puits, forage

\section{Introduction}

La ville de N'Djaména se situe à l'Ouest du Tchad à $15^{\circ} 02$ de longitude Est et à $12^{\circ} 07$ de latitude Nord, et se trouve à une altitude variant entre 294 et 298 m (figure 1). Elle fait partie du bassin du Lac Tchad qui est le plus grand bassin endoréique mondial avec une superficie de 2500000 $\mathrm{Km}^{2}$. Elle est soumise à un climat de type sahélien, caractérisé par une courte période de pluie et saison sèche plus longue, la durée minimum de précipitation est de quatre mois (de juin à septembre) tandis que la durée maximum est de huit mois (avril à novembre). Les précipitations moyennes annuelles sont de l'ordre de $540 \mathrm{~mm}$. La température moyenne calculée sur 10 ans d'observation à partir des données provenant de la Direction des Ressources en Eau et de la Météorologie s'élève à environ $28^{\circ} \mathrm{C}$, avec un minimum mensuel de $23,4^{\circ} \mathrm{C}$ et un maximum mensuel de $33,5^{\circ} \mathrm{C}$.

La ville de N'Djaména est située dans une plaine alluviale très plate longée sur toute sa bordure sud par le fleuve Chari (figure 1). D'après la littérature (Gac, 1980 ; Olivry, 1996), le fleuve Chari, le plus important du Tchad, prend sa source dans le mont Yadé (en Centrafrique). Il est rejoint par son principal affluent, le Logone, au niveau de la ville. Ce dernier prend sa source dans le massif de l'Adamaoua au Cameroun. 
A travers la ville de N'Djaména, on note la présence de dépressions qui correspondent à des carrières creusées pour les constructions des maisons. Pendant la saison des pluies, les eaux de pluie et de ruissellement s'accumulent dans ces dépressions ; elles y stagnent durant toute la période humide avant de se dissiper soit par évaporation soit par infiltration, ou encore sous l'effet conjugué des deux phénomènes donnant ainsi naissance à des mares plus ou moins permanentes selon leur importance. Il y a également la présence d'un grand canal qui traverse la ville du nord au sud et qui a pour but de drainer les eaux usées.

Le sous-sol de la ville de N'Djamena renferme, dans son ensemble, deux niveaux aquifères superposés (Schneider et Wollf, 1992 , BRGM, 1988, Kushnir, 1993, IWAKO, 1985). Ces derniers sont séparés par un niveau argileux imperméable à semi-perméable par endroit ; ces deux aquifères font plutôt partie d'un même ensemble, car c'est la sédimentation sableuse marquée par les dépôts de lentilles argileuses qui a permis leur différentiation. Par ailleurs, le BRGM (1967) signale également localement, la présence d'aquifères intermédiaires vers 20 à $30 \mathrm{~m}$ de profondeur. L'aquifère quaternaire est donc un aquifère multicouche.

La nappe superficielle est captée par les puits ouvert tandis que la nappe sous jacente est captée par les pompes manuelles. Ces nappes sont alimentées principalement par le fleuve Chari et quelque fois par les eaux de pluie (Kadjangaba 2007 ; Kadjangaba et al, 2017).

La ville de N'Djaména, à l'instar de toutes les grandes cités d'Afrique, connait également une croissance rapide de sa population, Cela peut entrainer des risques de contamination des eaux souterraines, liés aux activités humaines. Ce risque diminue la disponibilité de l'eau, c'est ainsi que pour assurer sa pérennité ainsi que sa bonne gestion, dans le cadre du développement durable, il est judicieux de faire un suivit permanent de la qualité physico-chimique des eaux.

Comme toute ville urbaine, la minéralisation des eaux peut résulter de l'interaction de plusieurs processus hydro chimiques. Ainsi l'objectif de ce travail est d'évaluer l'impact du processus hydro chimique sur la qualité des eaux souterraines de la ville de N'Djaména. 


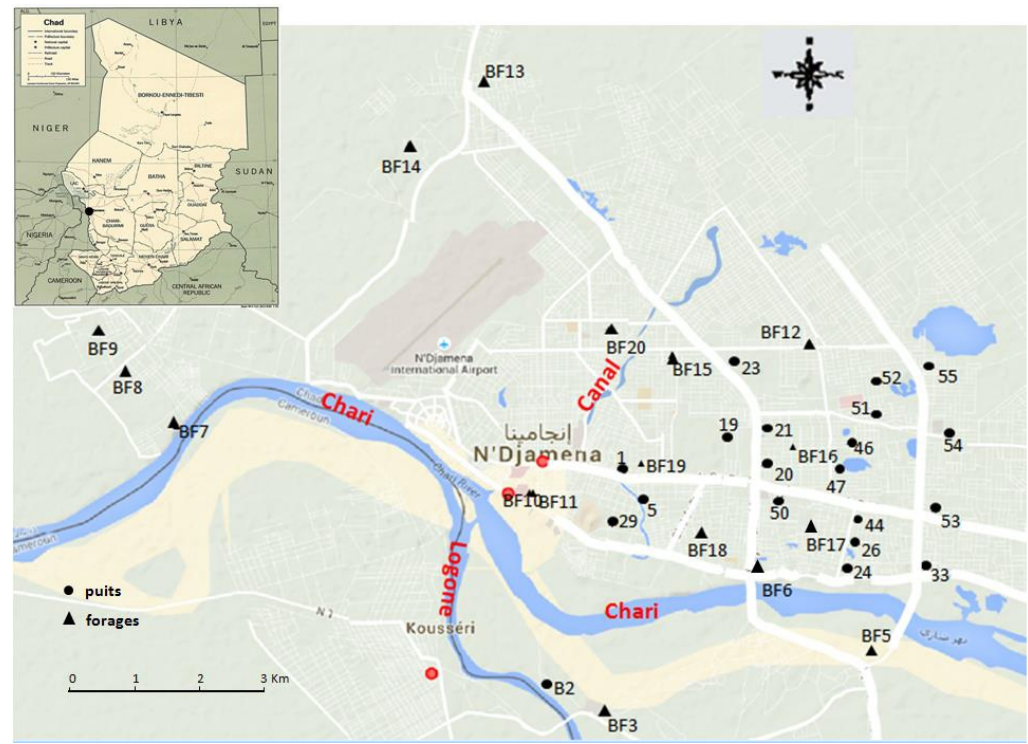

Figure 1 : Localisation de la zone d'étude et des points échantillonnés

\section{Echantillonnage, analyse et méthode}

Pour réaliser cette étude, nous avons prélevé de manière aléatoire, au total 38 points d'eaux souterraines réparti à travers la ville (figure 1), parmi lesquelles on a 20 points d'eaux provenant de la nappe superficielle située à environ $10 \mathrm{~m}$ de profondeur, prélevé durant les mois de mai, septembre et novembre 2003 et le mois d'août 2004 et 16 points d'eaux de la nappe sous jacente située entre 30 et $40 \mathrm{~m}$ de profondeur, prélevés au mois d'août 2010 et 2 points du fleuve Chari en août 2010 . .

Le pH et la température ont été mesurés in situ grâce à l'électrode mixte pH 323/Set B et la conductivité grâce à l'électrode Lf 318/Set. Il en est de même pour l'alcalinité qui est déterminée par titrimétrie, à l'aide d'un titrateur digital de marque $\mathrm{HACH} / 16900$.

Les échantillons destinés aux analyses de laboratoire ont été conservés dans des flacons en polyéthylène après avoir été filtrés avec des filtres en cellulose de $0,45 \mu \mathrm{m}$ et acidifiés pour les cations. L'analyse des ions majeurs a été réalisée au Laboratoire d'Hydrogéologie de l'Université d'Avignon. les anions $\left(\mathrm{Cl}^{-}, \mathrm{SO}_{4}{ }^{2-}, \mathrm{NO}_{3}{ }^{-}\right)$ont été analysés à l'aide d'un Chromatographe ionique Dionex avec une incertitude relative de $3 \%$, et les cations $\left(\mathrm{Ca}^{2+}, \mathrm{Mg}^{2+}\right.$, $\mathrm{Na}^{+}, \mathrm{K}^{+}$) à l'aide d'un spectromètre d'absorption atomique pour avec une incertitude relative de $5 \%$.

Pour traiter les résultats des analyses, nous avons utilisé le diagramme de piper afin d'identifier les différents types d'eaux souterraines et de définir les processus hydrochimiques plausibles qui régissent la chimie de ces eaux et l'Analyse en Composantes Principale (ACP) qui est une méthode d'analyse statistique descriptive de données multidimensionnelles. Elle a pour but 
d'établir une relation entre plusieurs variables chimiques et de regrouper celles qui présentent un comportement semblable. Cette méthodologie est largement utilisée pour interpréter les données hydrochimiques dans le domaine des sciences de l'eau, pour l'étude des phénomènes qui sont à l'origine de la minéralisation des eaux.

\section{Paramètres physico-chimiques}

Les résultats des paramètres physico-chimiques des eaux souterraines de la ville de N'Djaména sont récapitulés dans le tableau 1. D'une manière générale, ils sont très variables.

La mesure de la conductivité permet d'évaluer rapidement mais très approximativement la minéralisation globale de l'eau et d'en suivre l'évolution (Rodier et al, 2009). Les valeurs de la conductivité de notre zone d'étude sont très hétérogènes et sont comprises entre 531,45 et $1253 \mu \mathrm{S} / \mathrm{Cm}$ (avec une moyenne $271 \mu \mathrm{S} / \mathrm{Cm}$ ) pour les eaux captées par les puits et entre 71 et 596,00 (avec une moyenne $253,13 \mu \mathrm{S} / \mathrm{Cm}$ ) pour les eaux captées par les forages 'tableau 2). Du point de vue spatiale, la répartition des conductivités est très hétérogène, cela pourrait être liées aux diverses sources de minéralisation des eaux.

La température des eaux souterraines est presque homogène (tableau 2). En effet, elle varie entre $29,6^{\circ} \mathrm{C}$ et $31,8^{\circ} \mathrm{C}$ avec une moyenne de $30,59^{\circ} \mathrm{C}$ dans les puits et entre $28,9^{\circ} \mathrm{C}$ et $31,5^{\circ} \mathrm{C}$ avec une moyenne de $30,12^{\circ} \mathrm{C}$ dans les forages. Ces valeurs reflètent celle de la température de l'air ambiant dont la moyenne est de $28^{\circ} \mathrm{C}$ dans notre zone d'étude. Cela laisse penser que le système aquifère du Quaternaire est en équilibre thermique avec l'atmosphère.

Le pH mesuré quant à lui varie entre 6,13 et 7,58 avec une moyenne de 6,85 dans les puits et entre 6,70 et 7,71 avec une moyenne de 7,24 dans les forages.

La distribution statistique des ions majeurs (figure 2 et 3) donne les proportions des différents éléments présents dans l'eau. Les échantillons dans leur ensemble sont surtout dominés au niveau des anions par $\mathrm{HCO}_{3}{ }^{-}$qui représente $74 \%$ et $94 \%$ de la totalité des anions dans les puits et forages avec une moyenne respective de 205,4 $\mathrm{mg} / \mathrm{l}$ et 143,06 $\mathrm{mg} / \mathrm{l}$ ( tableau 2). Les teneurs en anions tels que les ions $\mathrm{Cl}^{-}(11 \%)$ ont une moyenne de 29,79 mg/l suivi des ions $\mathrm{NO}_{3}{ }^{-}(10 \%)$ avec une moyenne de $28,30 \mathrm{mg} / \mathrm{l}$ puis des ions $\mathrm{SO}_{4}{ }^{2-}(5$ $\%$ ) avec une moyenne de 14,71 mg/l dans les puits, dépassent largement les teneurs qu'on trouve dans les forages. Ces dernières sont faibles à nulles (entre 0 et $3 \%$ ).

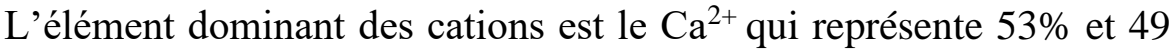
$\%$ de la totalité des cations avec des moyennes respectives de 50,27 et 26,79 $\mathrm{mg} / \mathrm{l}$ dans les puits et forages. Il est suivit $\mathrm{du} \mathrm{Na}^{+}$qui représentent respectivement $34 \%$ et $24 \%$ de la totalité des cations des puits et forages avec 
des teneurs de 34,47 mg/l et 11,84 mg/l. Ces deux ions sont suivit du $\mathrm{Mg}^{2+}$ avec une moyenne de 8,83 et $7,03 \mathrm{mg} / 1$ soit $9 \%$ et $14 \%$ de l'ensemble des cations et du $\mathrm{K}^{+}$avec une moyenne de $8,01 \mathrm{mg} / \mathrm{l}$ dans les puits et $4,67 \mathrm{mg} / \mathrm{l}$ dans les forages, ce qui représentent $8 \%$ et $9 \%$ de l'ensemble des cations.

Tableau 1 : Les données physico-chimiques des eaux souterraines.

\begin{tabular}{|c|c|c|c|c|c|c|c|c|c|c|c|c|}
\hline Date & $\begin{array}{c}\text { Nom des } \\
\text { échantillons }\end{array}$ & $\begin{array}{c}\text { Type } \\
\text { d'ouvrage }\end{array}$ & $\mathrm{pH}$ & $\mathrm{c} 25^{\circ} \mathrm{C}$ & $\mathrm{HCO}_{3}^{-}(\mathrm{mg} / \mathrm{l})$ & $\mathrm{Cl}(\mathrm{mg} / \mathrm{l})$ & $\mathrm{NO}_{3}^{-}(\mathrm{mg} / \mathrm{l})$ & $\mathrm{SO}_{4}^{--}(\mathrm{mg} / \mathrm{l})$ & $\mathrm{Ca}^{++}(\mathrm{mg} / \mathrm{l})$ & $\mathrm{Mg}^{++}(\mathrm{mg} / \mathrm{l})$ & $\mathrm{Na}^{+}(\mathrm{mg} / \mathrm{l})$ & $\mathrm{K}^{+}(\mathrm{mg} / \mathrm{l})$ \\
\hline $28 / 05 / 2003$ & 19 & puits & 7,58 & 345,00 & 148,80 & 5,88 & 11,00 & & 21,64 & 0,47 & 40,25 & 3,53 \\
\hline $28 / 05 / 2003$ & 20 & puits & 7,19 & 449,00 & 194,40 & 11,47 & 15,40 & 6,62 & 18,63 & 11,94 & 32,39 & 6,94 \\
\hline $28 / 05 / 2003$ & 21 & puits & 7,30 & 309,00 & 168,00 & 5,29 & 4,40 & 6,43 & 26,64 & 4,34 & 24,15 & 5,48 \\
\hline $28 / 05 / 2003$ & 23 & puits & 7,16 & 533,00 & 181,00 & 29,62 & 17,60 & 8,72 & 32,06 & 2,81 & 40,98 & 5,10 \\
\hline $27 / 05 / 2003$ & 33 & puits & 6,82 & 330,00 & 127,00 & 10,00 & 0,00 & 6,28 & 27,42 & 3,32 & 6,12 & 5,10 \\
\hline $27 / 05 / 2003$ & 44 & puits & 6,70 & 370,00 & 130,0 & 12,43 & 8,80 & 14,77 & 20,94 & 6,93 & 19,10 & 6,62 \\
\hline $27 / 05 / 2003$ & 46 & puits & 6,97 & 392,00 & 169,00 & 4,71 & 2,20 & 3,28 & 32,87 & 4,68 & 11,21 & 4,68 \\
\hline $23 / 09 / 2003$ & 33 & puits & 6,78 & 337,00 & 189,00 & 1,10 & 1,45 & 0,12 & 43,65 & 2,70 & 15,09 & 2,93 \\
\hline 23/09/2003 & 44 & puits & 6,45 & 411,00 & 144,00 & 14,82 & 13,47 & 14,64 & 28,10 & 6,50 & 23,86 & 5,60 \\
\hline $23 / 09 / 2003$ & 46 & puits & 6,78 & 385,00 & 211,20 & 3,68 & 0,89 & 1,48 & 40,75 & 9,32 & 18,72 & 4,40 \\
\hline $23 / 09 / 2003$ & 51 & puits & 6,61 & 347,00 & 180,00 & 5,19 & 0,10 & 2,08 & 32,90 & 6,67 & 21,21 & 7,38 \\
\hline $23 / 09 / 2003$ & 52 & puits & 6,49 & 312,00 & 120,00 & 9,58 & 29,35 & 6,28 & 24,52 & 5,68 & 23,13 & 13,22 \\
\hline $23 / 09 / 2003$ & 53 & puits & 6,13 & 286,00 & 152,00 & 17,00 & 15,81 & 9,46 & 34,81 & 4,20 & 40,32 & 3,38 \\
\hline $23 / 09 / 2003$ & 55 & puits & 6,57 & 282,00 & 160,80 & 1,05 & 1,92 & 1,49 & 29,51 & 9,32 & 11,12 & 4,39 \\
\hline $04 / 11 / 2003$ & 1 & puits & 6,55 & 578,00 & 252,00 & 33,67 & 8,57 & 19,89 & 68,90 & 6,54 & 49,78 & 7,25 \\
\hline $04 / 11 / 2003$ & 29 & puits & 6,76 & 406,00 & 199,20 & 14,09 & 1,77 & 6,79 & 45,51 & 10,80 & 18,25 & 7,82 \\
\hline $03 / 11 / 2003$ & 33 & puits & 6,85 & 384,00 & 218,40 & 3,15 & 7,59 & 0,83 & 67,10 & 4,76 & 7,87 & 2,23 \\
\hline $03 / 11 / 2003$ & 44 & puits & 6,63 & 359,00 & 139,20 & 15,40 & 25,30 & 14,85 & 38,69 & 6,72 & 20,39 & 4,87 \\
\hline $03 / 11 / 2003$ & 46 & puits & 6,99 & 367,00 & 204,00 & 4,15 & 0,27 & 2,00 & 54,10 & 9,20 & 8,68 & 4,37 \\
\hline $03 / 11 / 2003$ & 51 & puits & 6,78 & 406,00 & 223,20 & 6,60 & 2,07 & 1,04 & 40,56 & 8,46 & 24,37 & 8,58 \\
\hline $03 / 11 / 2003$ & 52 & puits & 6,52 & 292,00 & 122,40 & 8,66 & 23,89 & 5,91 & 22,46 & 4,75 & 22,99 & 12,69 \\
\hline 03/11/2003 & 54 & puits & 7,05 & 320,00 & 151,20 & 6,12 & 11,61 & 9,88 & 21,60 & 4,11 & 43,73 & 4,06 \\
\hline $03 / 11 / 2003$ & 55 & puits & 6,53 & 271,00 & 153,60 & 1,17 & 1,61 & 1,54 & 29,69 & 8,90 & 11,51 & 4,47 \\
\hline $10 / 08 / 2010$ & B2 & puits & 7,56 & 418,00 & 268,00 & 4,51 & 0 & 2,12 & 51,94 & 10,64 & 16,62 & 3,53 \\
\hline $27 / 05 / 2003$ & 47 & puits & 6,78 & 732,00 & 283,00 & 25,59 & 22,00 & 10,49 & 59,51 & 7,02 & 37,25 & 9,01 \\
\hline $27 / 05 / 2003$ & 50 & puits & 7,14 & 999,00 & 185,00 & 35,88 & 56,00 & 6,18 & 44,73 & 10,02 & 37,81 & 9,20 \\
\hline $23 / 09 / 2003$ & 47 & puits & 6,57 & 832,00 & 316,80 & 43,77 & 89,14 & 20,25 & 84,50 & 33,62 & 41,81 & 13,36 \\
\hline 23/09/2003 & 50 & puits & 6,99 & 983,00 & 282,40 & 48,03 & 284,70 & 15,46 & 75,47 & 20,24 & 78,66 & 46,34 \\
\hline $04 / 11 / 2003$ & 5 & puits & 7,13 & 1217,00 & 307,20 & 168,35 & 42,85 & 99,45 & 143,24 & 11,06 & 78,24 & 7,82 \\
\hline $03 / 11 / 2003$ & 47 & puits & 6,65 & 805,00 & 331,20 & 39,48 & 78,06 & 19,36 & 73,48 & 32,02 & 35,36 & 10,42 \\
\hline $03 / 11 / 2003$ & 53 & puits & 6,81 & 288,00 & 130,00 & 4,22 & 76,61 & 10,88 & 28,35 & 3,09 & 48,91 & 6,05 \\
\hline $28 / 03 / 2003$ & 1 & puits & 6,77 & 590,00 & 228,00 & 25,88 & 8,80 & 26,32 & 42,08 & 0,23 & 56,34 & 6,71 \\
\hline $28 / 03 / 2003$ & 5 & puits & 7,43 & 998,00 & 264,00 & 92,00 & 48,40 & 23,10 & 68,73 & 5,15 & 72,06 & 7,44 \\
\hline $04 / 11 / 2003$ & 52 & puits & 6,52 & 292,00 & 122,00 & 8,66 & 23,89 & 5,91 & 22,46 & 4,75 & 22,99 & 12,69 \\
\hline $04 / 11 / 2003$ & 23 & puits & 7,02 & 493,00 & 236,00 & 18,24 & 20,12 & 6,08 & 47,25 & 7,23 & 48,42 & 4,91 \\
\hline $04 / 11 / 2003$ & 5 & puits & 7,13 & 1217,00 & 307,00 & 168,35 & 42,85 & 99,45 & 143,24 & 11,06 & 78,24 & 7,82 \\
\hline $28 / 01 / 2004$ & 1 & puits & 6,75 & 561,00 & 257,60 & 32,45 & 0,53 & 19,39 & 69,30 & 6,04 & 48,49 & 7,55 \\
\hline $28 / 01 / 2004$ & 5 & puits & 7,3 & 1253,00 & 332,00 & 158,40 & 0,00 & 37,35 & 148,46 & 10,92 & 78,22 & 9,40 \\
\hline $28 / 01 / 2004$ & 23 & puits & 6,83 & 832,00 & 316,80 & 44,23 & 81,55 & 21,90 & 82,10 & 32,47 & 43,42 & 10,99 \\
\hline $28 / 01 / 2004$ & 47 & puits & 6,59 & 277,00 & 110,40 & 8,60 & 23,13 & 5,66 & 22,87 & 4,39 & 20,88 & 12,04 \\
\hline $10 / 08 / 2010$ & BF3 & forage & 7,06 & 308,00 & 186,00 & 2,62 & 0 & 4,42 & 29,47 & 7,83 & 21,06 & 4,53 \\
\hline $10 / 08 / 2010$ & BF5 & forage & 6,74 & 71,00 & 40,00 & 0,39 & 0,02 & 0,03 & 5,26 & 1,86 & 3,90 & 1,86 \\
\hline $10 / 08 / 2010$ & BF6 & forage & 7,41 & 205,00 & 122,00 & 0,51 & 0,06 & 0,01 & 25,53 & 5,83 & 3,86 & 3,41 \\
\hline $11 / 08 / 2010$ & BF8 & forage & 7,45 & 207,00 & 120,00 & 0,32 & 0,03 & 0,01 & 24,20 & 5,90 & 4,38 & 4,19 \\
\hline $11 / 08 / 2010$ & BF9 & forage & 6,90 & 176,00 & 116,00 & 0,38 & 0 & 0,21 & 15,38 & 4,34 & 10,97 & 4,36 \\
\hline $11 / 08 / 2010$ & BF10 & forage & 7,02 & 364,00 & 186,00 & 16,04 & 0,10 & 3,06 & 40,67 & 11,09 & 10,44 & 7,43 \\
\hline $11 / 08 / 2010$ & BF11 & forage & 7,04 & 312,00 & 158,00 & 12,91 & 0,02 & 0,40 & 36,56 & 9,18 & 6,09 & 4,09 \\
\hline $11 / 08 / 2010$ & $\mathrm{BF} 12$ & forage & 7,30 & 183,00 & 104,00 & 2,49 & 0,05 & 1,64 & 19,95 & 5,22 & 5,96 & 2,77 \\
\hline $11 / 08 / 2010$ & BF13 & forage & 6,70 & 259,00 & 120,00 & 2,77 & 0,07 & 20,58 & 19,99 & 6,90 & 16,49 & 5,32 \\
\hline $11 / 08 / 2010$ & BF14 & forage & 7,65 & 260,00 & 152,00 & 1,01 & 0 & 5,28 & 25,06 & 7,11 & 15,89 & 5,21 \\
\hline $12 / 08 / 2010$ & BF15 & forage & 7,54 & 433,00 & 258,00 & 3,19 & 0,04 & 13,94 & 52,29 & 12,29 & 20,36 & 8,06 \\
\hline $12 / 08 / 2010$ & BF16 & forage & 7,23 & 162,00 & 105,00 & 0,38 & 0 & 0,13 & 12,99 & 3,25 & 15,51 & 3,61 \\
\hline $12 / 08 / 2010$ & BF17 & forage & 7,60 & 105,00 & 61,00 & 1,59 & 0 & 0,05 & 11,07 & 2,75 & 5,26 & 2,81 \\
\hline $12 / 08 / 2010$ & BF18 & forage & 7,10 & 132,00 & 72,00 & 0,86 & 0 & 0,32 & 14,80 & 3,89 & 3,53 & 2,86 \\
\hline $12 / 08 / 2010$ & BF19 & forage & 7,71 & 277,00 & 164,00 & 2,56 & 1,09 & 1,87 & 21,53 & 4,35 & 29,84 & 4,77 \\
\hline $12 / 08 / 2010$ & BF20 & forage & 7,43 & 596,00 & 325,00 & 20,38 & 1,56 & 17,69 & 73,87 & 20,75 & 15,95 & 8,73 \\
\hline $10 / 08 / 2010$ & Chari & fleuve & 7.96 & 39,00 & 23,00 & 0.79 & 0 & 0.53 & 2.44 & 1.08 & 2.04 & 3.00 \\
\hline $11 / 08 / 2010$ & Chari & fleuve & 7.33 & 43,00 & 26,00 & 0.57 & 0 & 0.51 & 2.94 & 1.10 & 2.58 & 2.61 \\
\hline
\end{tabular}



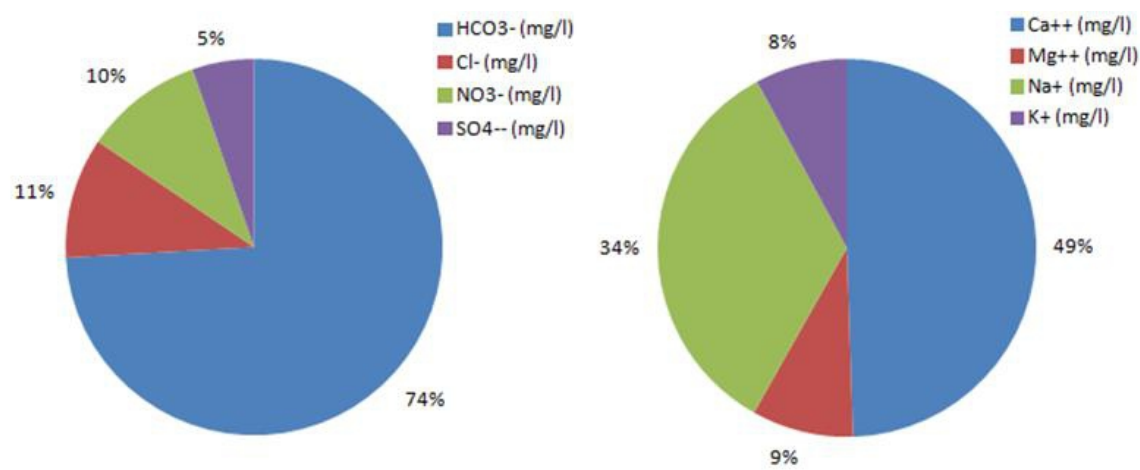

Figure 2 : Distribution statistique des anions et cations dans les puits.
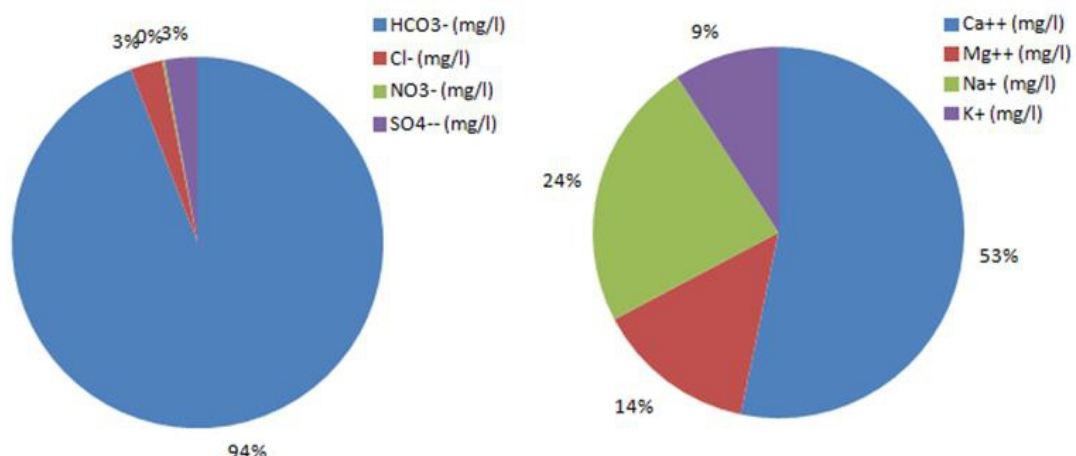

Figure 3 Distribution statistique des anions et cations dans les forages.

Tableau 2 : Résultats statistiques des mesures physico - chimiques

\begin{tabular}{|c|c|c|c|c|c|c|c|c|c|c|c|c|}
\hline \multirow{5}{*}{ Puits } & & $\mathrm{T}^{\circ} \mathrm{C}$ & $\mathrm{pH}$ & $\mathrm{c} 25^{\circ} \mathrm{C}$ & $\mathrm{HCO}-(\mathrm{mg} / \mathrm{l})$ & $\mathrm{Cl}-(\mathrm{mg} / \mathrm{l})$ & NO3- $(\mathrm{mg} / \mathrm{l}$ & SO4-- $(\mathrm{mg} / \mathrm{l})$ & $\mathrm{Ca}++(\mathrm{mg} / \mathrm{l})$ & $M g++(m g / l)$ & $\mathrm{Na}+(\mathrm{mg} / \mathrm{l})$ & $\mathrm{K}+(\mathrm{mg} / \mathrm{l})$ \\
\hline & $\min$ & 29,60 & 6,13 & 271,00 & 110,40 & 1,05 & 0,00 & 0,12 & 18,63 & 0,23 & 6,12 & 2,23 \\
\hline & $\max$ & 31,80 & 7,58 & 1253,00 & 332,00 & 168,35 & 284,70 & 99,45 & 148,46 & 33,62 & 78,66 & 46,34 \\
\hline & moy & 30,59 & 6,85 & 531,45 & 205,40 & 28,79 & 28,30 & 14,71 & 50,27 & 8,83 & 34,47 & 8,01 \\
\hline & Ecart-Type & 0,52 & 0,32 & 292,39 & 67,53 & 43,19 & 48,98 & 21,64 & 33,11 & 7,79 & 21,05 & 6,91 \\
\hline \multirow{4}{*}{ Forages } & $\min$ & 28,90 & 6,70 & 71,00 & 40,00 & 0,32 & 0,02 & 0,01 & 5,26 & 1,86 & 3,53 & 1,86 \\
\hline & $\max$ & 31,30 & 7,71 & 596,00 & 325,00 & 20,38 & 1,56 & 20,58 & 73,87 & 20,75 & 29,84 & 8,73 \\
\hline & moy & 30,12 & 7,24 & 253,13 & 143,06 & 4,28 & 0,30 & 4,35 & 26,79 & 7,03 & 11,84 & 4,63 \\
\hline & Ecart-type & 0,82 & 0,32 & 132,35 & 72,28 & 6,27 & 0,55 & 6,79 & 17,26 & 4,67 & 7,81 & 1,97 \\
\hline
\end{tabular}

\section{Les différents types d'eaux souterraines}

La représentation des concentrations des éléments majeurs sur le diagramme de piper (figure 4) réalisé grâce au logiciel DIAGRAMME (Simler, 2004), permet d'avoir une idée globale des différents types d'eaux rencontrée dans l'aquifère de la ville de N'Ndjamena.

Plusieurs types d'eaux ont été identifiés, on a ainsi :

- $\quad$ Les eaux de type bicarbonaté calcique correspondant essentiellement aux eaux du fleuve Chari, eaux prélevées dans les forages et une partie des eaux de puits. Ces eaux sont caractérisées par une conductivité relativement faible (Conductivité situé entre 39 et $43 \mu \mathrm{S} / \mathrm{Cm}$ pour le Fleuve Chari et ne dépassant pas $596 \mu \mathrm{S} / \mathrm{Cm}$ pour les eaux souterraines). Cependant, quelques 
échantillons de ce groupe possèdent des teneurs relativement élevées en nitrate et Chlorure. Ces derniers se trouvent à proximité du canal (1), des mares (échantillon 44) ou sont diversement repartis (échantillons 23, 47 : voir carte de localisation des points, figure 1).Cela concerne essentiellement les eaux de puits.

- $\quad$ Les eaux de type bicarbonaté sodique et potassique. On y trouve des points diversement repartis (échantillons 53, 54 : voir carte de localisation des points), la présence de $\mathrm{NO}^{-}$est quasi systématique.

- $\quad$ Les eaux de type chlorurées et nitratées calciques ou sodique dans lesquelles le bicarbonate reste dominant. Les échantillons concernés par ce type d'eau se trouvent à proximité du canal (échantillon 5) ou sont diversement repartis (échantillons 50 : voir carte de localisation des points, figure 1).

Les deux derniers types d'eaux regroupent essentiellement les eaux de puits et sont relativement plus minéralisées (les conductivités peuvent atteindre $1253 \mu \mathrm{S} / \mathrm{Cm}$ ). La présence des nitrates et chlorure en leur sein et dans une partie du premier type d'eau suggère un contact d'eau douce avec une source de contamination de surface telle que les eaux usées, les dépôts d'ordures ou encore une autre source comme les latrines riche en $\mathrm{Cl}^{-}$et $\mathrm{NO}_{3}{ }^{-}$. Les teneurs des ions $\mathrm{Cl}^{-}$et $\mathrm{NO}_{3}{ }^{-}$sont relativement élevés dans ces eaux et peuvent atteindre des valeurs maximales respectives de 168,35 et $284,70 \mathrm{mg} / \mathrm{l}$ (tableau 2), ces valeurs comparables à celle obtenues par Minda en 2016 (respectivement $201 \mathrm{mg} / \mathrm{l}$ et $217 \mathrm{mg} / \mathrm{l}$ de chlorure et nitrate) dans les périmètres irriguées de la même zone d'étude. Ceci fournit des indices quant à la présence d'une contamination anthropique des eaux souterraines corroborant ainsi notre hypothèse.

La présence des indices de contamination dans les eaux souterraines en milieu urbain est fréquent et a été également souligné dans d'autre ville sub saharienne comme Ouagadougou (Yameogo Ouandaogo, 2008) ou encore Niamey (Boubakar Hassane, 2010), et la principale source de pollution est dues essentiellement aux activités domestiques.

L'hétérogénéité de la composition chimique des eaux des puits peut être due aux nombreux processus qui interviennent dans la minéralisation de l'eau. 


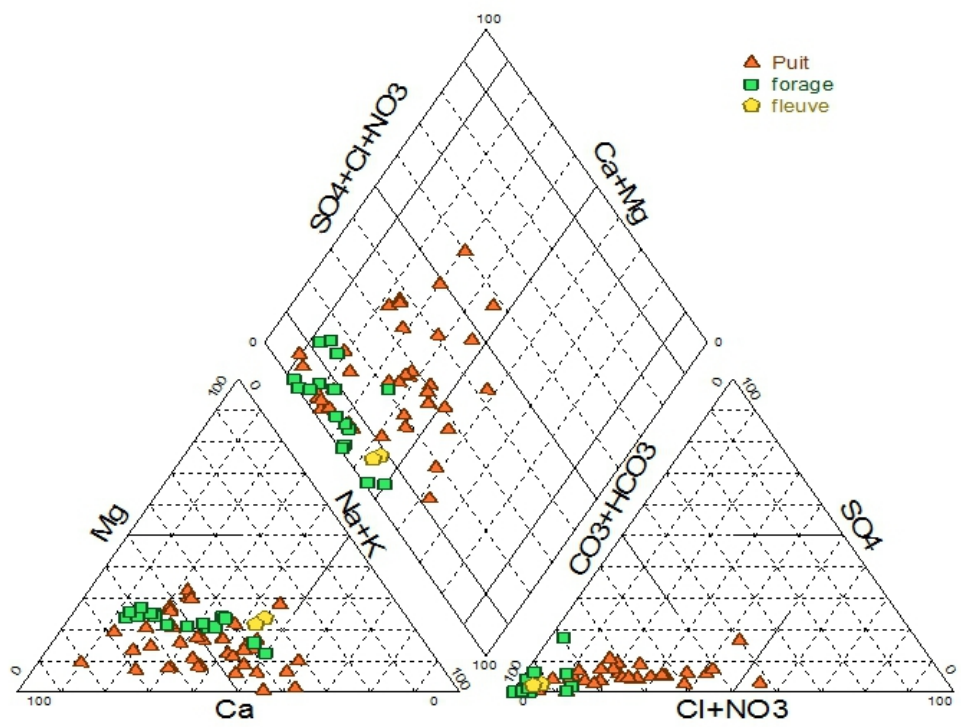

Figure 4 : Diagramme de piper

\section{Analyse statistique}

L'analyse statistique est une méthode couramment utilisée, depuis quelques années, dans de nombreuses études à travers le monde (Mudry et Blavoux ,1986; Reghunath et al, 2002 Matini et al, 2009; Hamzaoui et al, 2012; Askri, 2015) pour ne citer que celles là, et au Tchad (Djoret, 2000, Kadjangaba, 2007, Bande, 2016; Mondodjo, 2016) afin de mieux comprendre les processus hydro chimiques qui régissent la minéralisation globale des eaux souterraines.

Grace au logiciel IBM SPSS Statistics 21, une analyse factorielle des différents constituants chimiques des eaux souterraines de la ville de N'Djaména a été réalisée en prenant en compte la conductivité électrique, le $\mathrm{pH}$, tous les cations et anions. Les valeurs propres, le pourcentage de variance et le pourcentage cumulé de variance des facteurs sont donnés dans le tableau 3. La première valeur propre est de 5,63 , ce qui représente $56,29 \%$ de la variance totale et ceci constitue le premier et principal facteur. Les deuxième et troisième valeurs propres sont de 1,74 et 0,99 et représentent respectivement $17,42 \%$ et $9,94 \%$ de la variance totale. Les trois facteurs (F1, F2, F3) du tableau 3 traduisent l'essentiel des informations recherchées et permettent de représenter de manière significative le nuage de points car la somme de la variance exprimée par ces facteurs est de $83,65 \%$. 
Tableau 3 : Matrice du poids des différents ions dans les eaux souterraines de la ville de N'Djaména.

\begin{tabular}{|l|c|c|c|}
\hline & Facteur 1 & Facteur 2 & Facteur 3 \\
\hline $\mathrm{Clmg} / \mathrm{l}$ & $\mathbf{0 , 9 6}$ & 0,08 & 0,04 \\
\hline $\mathrm{SO} 4 \mathrm{mg} / \mathrm{l}$ & $\mathbf{0 , 9 2}$ & 0,00 & $-0,05$ \\
\hline $\mathrm{Ca} \mathrm{mg/l}$ & $\mathbf{0 , 9 0}$ & 0,25 & 0,20 \\
\hline $\mathrm{c} 25 \mathrm{C}$ & $\mathbf{0 , 8 7}$ & 0,40 & 0,12 \\
\hline $\mathrm{Na} \mathrm{mg/l}$ & $\mathbf{0 , 8 2}$ & 0,34 & $-0,16$ \\
\hline $\mathrm{HCO} \mathrm{mg} / \mathrm{l}$ & 0,68 & 0,46 & 0,36 \\
\hline $\mathrm{NO} 3 \mathrm{mg} / \mathrm{l}$ & 0,20 & $\mathbf{0 , 8 9}$ & $-0,19$ \\
\hline $\mathrm{K} \mathrm{mg} / \mathrm{l}$ & 0,15 & $\mathbf{0 , 8 8}$ & $-0,22$ \\
\hline $\mathrm{Mg} \mathrm{mg} / \mathrm{l}$ & 0,23 & $\mathbf{0 , 7 2}$ & 0,35 \\
\hline $\mathrm{pH}$ & 0,03 & $-0,13$ & $\mathbf{0 , 8 2}$ \\
\hline valeurs propres & 5,63 & 1,74 & 0,99 \\
\hline$\%$ de la variance & 56,29 & 17,42 & 9,94 \\
\hline \% de la variance cumulée & 56,29 & 73,71 & 83,65 \\
\hline
\end{tabular}

La contribution des différentes variables dans la définition des principaux facteurs est donnée par le tableau 3. Chaque facteur est défini par un certain nombre de variables essentielles dans la mise en évidence du mécanisme de minéralisation des eaux. Le premier facteur F1 (qui représente $56,29 \%$ du total de la variance) regroupe la conductivité électrique, le Calcium, les Chlorures, les sulfates, le sodium et l'alcalinité. Ce facteur apparaît comme un axe de minéralisation globale de l'eau. Les cinq éléments chimiques qui définissent le facteur $\mathrm{F} 1$, présentent des corrélations plus ou moins significatif entre eux (tableau 4). C'est ainsi qu'on a une bonne corrélation entre l'alcalinité et le calcium, et une corrélation plus modérée avec les sulfates, sodium et Chlorures. Ce facteur regroupe la plupart des éléments chimiques d'origine naturelle.

Les ions $\mathrm{Ca}^{2+}, \mathrm{Na}^{+}$et $\mathrm{HCO}_{3}{ }^{-}$, sont en générale mis en solution par hydrolyse des silicates (Njitchoua et al.,1997 ; Njitchoua et Ngounou, 1997, Djoret, 2000) . En effet, en traversant la zone non saturée, l'eau d'infiltration se charge en $\mathrm{CO}_{2}$ libéré par les activités biologiques, cette eaux riche en $\mathrm{CO}_{2}$ a la possibilité d'attaquer les minéraux allumino-silicatés. La formation de Kaolinite néoformée est produite lors de l'interaction eau-roche, ce processus est ainsi à l'origine de la libération des cations et des bicarbonates dans l'eau. La bonne relation qui existe entre la conductivité et l'alcalinité $(0,83)$ des eaux (tableau 4) montre que ce sont bien les bicarbonates qui président à la mise en solution des constituants matriciels (Ketchemen, 1992). La mise en solution des éléments peut également être liée aux échanges de cations au niveau des horizons argileux. 
Le tableau 4, montre qu' on a une bonne corrélation entre conductivité électrique et les chlorures reflétant ainsi leur rôle non négligeable dans la minéralisation globale des eaux. Dans les forages, les teneurs en chlorure sont faibles mais elles peuvent être assez élevées dans les puits (tableau 1). Comparé à la moyenne pondérée des chlorures qui est de $0,45 \mathrm{mg} / \mathrm{l}$ dans les pluies (Kadjangaba, 2007) et sachant qu'il n'y a pas de réservoirs géologiques de chlorure dans notre zone d'étude; ces valeurs élevées peuvent être dues à un apport anthropique couplé à l'effet de l'évaporation (Kadjangaba, 2007, Djebebe-Ndjiguim et al, 2013).

Tableau 4 : Matrice de corrélation des eaux souterraine de la ville de N’Djaména.

\begin{tabular}{|l|c|c|c|c|c|c|c|c|c|c|}
\hline & $\mathrm{pH}$ & $\mathrm{c} 25 \mathrm{C}$ & $\mathrm{HCO} 3 \mathrm{mg} / \mathrm{C}$ & $\mathrm{Clmg} / \mathrm{NO} \mathrm{mg} / \mathrm{SO}$ mg/ & $\mathrm{Ca} \mathrm{mg} /$ & $\mathrm{Mg} \mathrm{mg} /$ & $\mathrm{Na} \mathrm{mg} / \mathrm{l}$ & $\mathrm{K} \mathrm{mg} / \mathrm{l}$ \\
\hline $\mathrm{pH}$ & 1,00 & & & & & & & & & \\
$\mathrm{c} 25 \mathrm{C}$ & 0,07 & 1,00 & & & & & & & & \\
$\mathrm{HCO} 3$ & & & & & & & & & \\
$\mathrm{mg} / \mathrm{l}$ & 0,09 & $\mathbf{0 , 8 3}$ & 1,00 & & & & & & & \\
$\mathrm{Clmg} / \mathrm{l}$ & 0,11 & $\mathbf{0 , 8 8}$ & 0,62 & 1,00 & & & & & & \\
$\mathrm{NO} 3 \mathrm{mg} / \mathrm{l}$ & $-0,11$ & 0,50 & 0,36 & 0,28 & 1,00 & & & & & \\
$\mathrm{SO} 4 \mathrm{mg} / \mathrm{l}$ & 0,03 & $\mathbf{0 , 7 4}$ & 0,54 & $\mathbf{0 , 8 9}$ & 0,23 & 1,00 & & & & \\
$\mathrm{Ca} \mathrm{mg} / \mathrm{l}$ & 0,07 & $\mathbf{0 , 9 0}$ & $\mathbf{0 , 8 5}$ & $\mathbf{0 , 8 9}$ & 0,32 & 0,80 & 1,00 & & & \\
$\mathrm{Mg} \mathrm{mg} / \mathrm{l}$ & $-0,05$ & 0,51 & 0,67 & 0,27 & 0,51 & 0,24 & 0,49 & 1,00 & & \\
$\mathrm{Na} \mathrm{mg} / \mathrm{l}$ & $-0,02$ & $\mathbf{0 , 8 4}$ & 0,65 & $\mathbf{0 , 8 0}$ & 0,55 & 0,71 & 0,72 & 0,25 & 1,00 & \\
$\mathrm{~K} \mathrm{mg} / \mathrm{l}$ & $-0,13$ & 0,45 & 0,35 & 0,24 & $\mathbf{0 , 8 9}$ & 0,16 & 0,29 & 0,43 & 0,48 & 1,00 \\
\hline
\end{tabular}

Le facteur F2 (17,42\%) regroupe les ions $\mathrm{NO}_{3}{ }^{-}, \mathrm{K}^{+}$et $\mathrm{Mg}^{2+}$. Dans le contexte qui est le notre la présence des nitrates dans les eaux serait due à une pollution anthropique ( Njitchoua et al.,1997. Girard et Hillaire-Marcel., 1996 ; Djoret et Travi, 2001, Stadler et al, 2008, Foster et al 2011, Minda, 2016). Ce facteur met donc en évidence les échanges superficiels qui se font entre les eaux souterraines de la ville de N'Djaména et les eaux de ruissellement issues des précipitations et du drainage des sols en période de hautes eaux.

Dans le tableau 4, les ions sodium présentent une corrélation modérée avec les nitrates $(0,55)$ suggérant ainsi leur origine anthropique, du moins en partie. Ils présentent également une bonne corrélation avec les chlorures ce qui confirme l'origine anthropique des chlorures telle que nous l'avons souligné plus haut.

Le poids de l'alcalinité dans ce facteur n'est pas à négliger même s'il est plus faible que dans le premier facteur. Cela souligne le lien qu'il y a entre les ions $\mathrm{HCO}_{3}{ }^{-}$et $\mathrm{NO}_{3}{ }^{-}$tel que cela a déjà été observé lors d'études antérieures (kadjangaba, 2007). Le comportement de ces deux ions indique que l'introduction des ions $\mathrm{HCO}_{3}^{-}$dans le système peut résulter d'une transformation des ions $\mathrm{NO}_{3}^{-}$par un processus de dénitrification suivant l'équation : 


$$
5 \mathrm{C}+4 \mathrm{NO}_{3}{ }^{-}+2 \mathrm{H}_{2} \mathrm{O} \quad 2 \mathrm{~N} 2+4 \mathrm{HCO}_{3}{ }^{-}+\mathrm{CO} 2 \quad \text { (Kolle et al, 1983) }
$$

Le troisième facteur $\mathrm{F} 3(9,94 \%)$ est représenté par le pH, même si la corrélation entre cet élément et $\mathrm{NO}_{3}^{-}$(tableau 4) n'est pas très forte $(-0,11)$, l'opposition entre les deux éléments peut être liée à la présence du phénomène de dénitrification au sein de la nappe.

\section{Conclusion}

Ce travail a permis d'identifier trois principaux types d'eaux dans notre zone d'étude, à savoir :

- Les eaux de type bicarbonaté calcique, constitué par la majorité des échantillons. Ces derniers présentent des conductivités relativement faibles et regroupent aussi bien les eaux de puits que ceux de forages. Dans ce type d'eau, certains échantillons, surtout ceux provenant des puits ont des teneurs en nitrate assez élevées.

- Les eaux de type bicarbonaté sodique et potassique avec une présence de $\mathrm{NO}_{3}{ }^{-}$quasi systématique.

- Les eaux de type chlorurées et nitratées calciques ou sodique dans lesquelles le bicarbonate reste dominant.

Ces deux derniers types d'eaux peuvent avoir des conductivités qui peuvent parfois être relativement élevés, surtout dans les puits. Cette augmentation de conductivités s'accompagne d'augmentation des ions $\mathrm{Cl}^{-}$et $\mathrm{NO}_{3}{ }^{-}$dans les eaux.

En outre, l'analyse factorielle et la matrice de corrélation nous ont permis d'identifier les divers processus qui régissent la minéralisation globale des eaux souterraines de notre zone d'étude. Les résultats suggèrent donc, que la chimie des eaux souterraines est principalement influencée par l'hydrolyse des silicates, l'échange de cations entre les eaux souterraines et les minéraux argileux, l'évaporation et l'impact des activités anthropiques.

Comparée à la nappe superficielle qui présente des indice de contamination, la nappe sous jacente parait être pour l'instant protégé mais il ne faut pas exclure le fait qu'elle puisse avoir des problèmes en cas de surpompage de la nappe pouvant drainer la nappe superficielle.

\section{References:}

1. Askri B., Hydrochemical processes regulating groundwater quality in the coastal plain of Al Musanaah, Sultanate of Oman. Journal of African Earth Sciences N 106 pp : 87-98. 2015.

2. Bande F., Caractérisation hydrodynamique et hydrochimique des eaux souterraines en contexte urbain : cas de la ville de Moundou (Sud-ouest du Tchad). Mémoire de Master, Université de N'gaoundéré.65 p. 2016. 
3. Boubakar Hassane A. Aquiferes superficiels et profonds et pollution urbaine en afrique : Cas de la communauté urbaine de Niamey (NIGER). Thèse de l'Université Abdou Moumouni de Niamey.198 p. 2010.

4. BRGM, Etude de la vulnérabilité des nappes aquifères superficielles de N'Djaména (Tchad) et recommandations pour les aménagements. 90 p. 1988.

5. Djebebe-Ndjiguim C.L., Huneau F., Denis A., Foto E., Moloto-aKenguemba G., Celle Jeanton H. , Garel E. , Jaunat J., Mabingui J.and Le Coustumer P., Characterization of the aquifers of the Bangui urban area, Central African Republic, as an alternative drinking water supply resource.Hydrological Sciences Journal $N^{\circ} 58$ (8) pp : 1760-1778. 2013.

6. Djoret D., Etude de la recharge de la nappe du Chari Baguirmi par des méthodes chimiques et isotopiques. Thèse de l'Université d'Avignon des pays de Vaucluse (France). 161p. 2000.

7. Djoret D.and travi Y., Groundwater vulnerability and recharge or paleorecharge in the southeastern Chad basin, Chari Baguirmi aquifer. In: Isotope techniques in water resource investigations in arid and semi-arid regions, -IAEA TECDOC1207, pp : 33-40. 2001.

8. Foster S.D., Hirata R., and Howard K.W.F., Groundwater use in developing cities: policy issues arising from current trends.Hydrogeology Journal $\mathrm{N}^{\circ} 19$ pp : 271-274. 2011.

9. Gac J.Y., Géochimie du bassin du lac-Tchad. Travaux et documents de l'O.R.S.T.O.M. n 1243, 251 p. 1980.

10. Girard, P. and Hillaire-Marcel, C., 1996. Determining the source of nitrate pollution in Niger discontinuous aquifers using the natural $15 \mathrm{~N} / 14 \mathrm{~N}$ ratios. Journal of Hydrology, 199, 3-4, pp.239-251.

11. Hamzaoui A.F., Bouhlila R. et Gueddari M., Caractérisation des eaux de la nappe des grès du Trias (Sud-Est Tunisien) par les méthodes géochimiques et statistiques. Geo-Eco-Trop. $\mathrm{N}^{\circ} 36$ pp : 49-62. 2012.

12. IWAKO, Les ressources en eaux du Tchad. 1985.

13. Kadjangaba E., Etude hydrochimique et isotopique du système zone non saturé - nappe dans la zone urbaine de N'Djamena : impact de la pollution. Thèse de l'Université d'Avignon et des pays de Vaucluse, 206p. 2007.

14. Kadjangaba E, Huneau F., Travi Y. and Djoret D. Recharge and Groundwater quality of an Alluvial Aquifer : Case of the City of N'djamena (Chad). Journal of Environmental Science and Engineering B6, p: 493 - 505. 2017.

15. Ketchemen B., Etude hydrogéologique du grand Yaéré (Extrême-nord Cameroun) Synthèse hydrogéologique et étude de la recharge par les 
isotopes de l'environnement. Thèse de l'Université Cheikh Anta Diop de Dakar, 216 p. 1992.

16. Kolle W., Warner P., Strebel O., and Bollcher J., Denitrification by pyrite in a reducting aquifer. Vom Wasser $\mathrm{N}^{\circ}$ 61(1) pp : 125 - 147. 1983.

17. Kushnir I., Géologie, ressources minérales et ressources en eau au Tchad. Travaux et documents scientifiques du Tchad. Connaissance du Tchad I. 100 p. 1993.

18. Matini L., Moutou J. M. et Kongo-Mantono M.S., Évaluation hydrochimique des eaux souterraines en milieu urbain au Sud-ouest de Brazzaville, Congo. Afrique Science $N^{\circ} 5$ (1) pp : 82-98. 2009.

19. Minda M. Evaluation de la qualité des eaux souterraines dans les zones irriguées de la ville de N'Djamena. Mémoire de Master, Université de N'gaoundéré.53p. 2016.

20. Mudry J. et Blavoux B., Utilisation de l'analyse en composantes principales (sur les variétés centre réduites) pour l'étude du fonctionnement hydro cinématique de trois aquifères karstiques du sud-est de la France. Hydrogéologie $\mathrm{N}^{\circ} 1, \mathrm{p}:$ : 53-59. 1986.

21. Njitchoua R., Dever L., Fontes J.C. and Naah E., Geochemistry, origin and recharge mechanisms of groundwaters from the GarouaSandstone aquifer, northern Cameroon. Journal of Hydrology $\mathrm{N}^{\circ} 190$ pp: 123140. 1996.

22. Njitchoua R. et Ngounou N., Hydrochemistry and environmental isotope investigation of North Diamare Plain, nothern Cameroon. Journal of African Earth Sciences Nº 25 (2) pp : 307-316. 1997.

23. Olivry J.C., Chouret A., Vuillaume G., Lemoelle J., Briquet J.P., Hydrologie du lac Tchad. Monographie hydrologique 12. ORSTOM, Paris. 1996.

24. Reghunath R, Sreedhara Murthy T.R., Raghavan B.R., The utility of multivariate statistical techniques in hydrogeochemical studies:an example from Karnataka, India. Water Research $N^{\circ} 36$ pp : 2437 2442. 2002.

25. Rodier J., Bernard L., Nicole M., L'analyse de l'eau, 9e édition. DUNOD (éditeur), Paris, France.1526p. 2009.

26. Simler, R., Diagrammes. Laboratoire d'Hydrogéologie d'Avignon,Université d'Avignon, Logiciel d'hydrochimie multilangage en distribution libre. 2004.

27. Stadler S., Osenbrück K., Knöller K., Suckow A., Sültenfub E. H., Himmelsbach, T. et Hötzl, H., Understanding the origin and fate of nitrate in groundwater of semi-arid environments. Journal of Arid Environments $\mathrm{N}^{\circ} 72 \mathrm{pp}: 1830-1842.2008$. 
28. Schneider J.L. and Wolff J.P., Carte géologique et Hydrogéologique au 1/1 500000 de la République du Tchad. Mémoire explicatif. Document BRGM n 209 vol. 1,2, 689 p. 1992.

29. Yameogo Ouandaogo S. Ressources en eau souterraine du centre urbain de Ouagadougou au Burkina Faso qualité et vulnérabilité. Thèse de l'Université d'Avignon et des Pays de Vaucluse 245 p. 2008. 